\title{
PER LA FORMAZIONE DI NUOVI CENTRI DI CULTURA TECNICA SUPERIORE.
}

\author{
Prof. Ing. GUSTAVO COLONNETTI.
}

Che una riforma dei nostri istituti di istruzione tecnica superiore si imponga, è ormai fuori di discussione.

Se ne parla da parecchi anni, ed i progetti di nuovi regolamenti, e le relazioni di commissioni competenti, ed i pareri del Consiglio snperiore dell'istruzione pubblica, si sono succednti, discordi bensì nell'apprezzamento dei provvedimenti da ritenersi più opportuni ed urgenti, ma concordi tutti nell'affermare la improrogabile necessità di una riforma.

Non io dunque mi dilungherò in uno studio critico degli istituti esistenti, tanto più che io apprezzo al più alto grado lo sforzo che essi hanno sempre fatto, e van tuttora facendo, per adattarsi alle esigenze sempre mutevoli della vita professionale e per secondarne i progressi; e credo fermamente che se, sotto qualche punto di vista, essi non giungono più a soddisfare tutte le nostre aspirazioni di tecnici e di studiosi, ciò dipende non tanto da una loro incapacità di evolversi, quanto dalla eccessiva rapidità con cui essi hanno dovuto e saputo evolversi; rapidita che non ha nessun riscontro, neppure approssimativo, nella evoluzione degli altri rami della nostra cultura superiore; che non fu prevista, nè poteva logicamente venir prevista, da chi questi nostri istituti aveva pur così lucidamente concepiti e genialmente attuati; e che perciò basta da sola a spiegare il perchè le scuole d'ingegneria, che pur sono le più giovani per età fra le istituzioni nostre universitarie, appaiono oggi a taluni già tanto invecchiate da richiedere che se ne rinnovino financo le fondamenta. 
Mi si consenta soltanto di rilevare, nello stato di fatto oggi esistente, un punto su cui la nostre scuole d'ingegneria non hanno mai saputo o potuto prendere una posizione netta e decisa: è quello che riguarda il giusto equilibrio fra l'indirizzo teorico, o scientifico che dir si voglia, e l'indirizzo pratico, o professionale.

Una continua incertezza regna al riguardo: sia che si tratti della separazione o meno del biennio di avviamento da quello della facoltà di scienze, sia the si tratti dello sviluppo da attribuirsi alle matematiche là dove tale separazione è ormai un fatto compiuto, sia infine che si tratti dell'indirizzo da darsi ai corsi del successivo triennio di applicazione, sempre il medesimo dilemma risorge immutato nell' eterno contrasto fra coloro che dal prevalere dei corsi di intonazione pratica sperano una più efficace preparazione dell'allievo alla vita professionale, e coloro che nella riduzione degli insegnamenti cosidetti teorici vedono il pericolo di un graduale abbassamento del livello intellettuale dei futuri ingegnerj.

Checchè vi sia di vero nella speranza degli uni e nel timore degli altri, è certo che il contrasto è ben lungi dall' esser sopito, e che il parere recentemente emesso dal Consiglio superiore dell'istruzione pubblica, relativamente ad una progettata riforma generale universitaria, to ha fatto rinascere più vivo che mai.

Nè io credo che un vero equilibrio potrà su questo punto. esser raggiunto fin che permane la duplicità degli scopi a cui le nostre scuole di ingegneria debbono oggi in qualche modo mirare.

Esse non sono infatti chiamate soltanto a preparare per la vita tecnica e professionale una massa cospicua di giovani $i$. quali sappiano, appena lasciate le aule scolastiche, prontamente orientarsi in una qualunque delle numerose carriere loro aperte nelle pubbliche amministrazioni e nelle industrie private, grazie ad un felice connubio di quelle nozioni teoriche che 
sono indispensabile substrato ad ogni attività tecnica, e di quella conoscenza del mondo fisico che sola permette di vedere ogni problema pratico nella sua giusta luce, e sovente di intuirne la soluzione prima ancora di averla trovata.

Questo è bensì indubbiamente lo scopo dichiarato, e più immediatamente appariscente, delle scuole di ingegneria.

Ma esse ne hanno oggi anche un altro, più nascosto $e$ delicato, e pur non meno importante: ed è quello di provvedere all'ulteriore sviluppo delle scienze tecniche indirizzando ad esse un numero assai più esiguo, ma più scelto, di giovani, affinchè non si affievolisca nel nostro Paese quella produzione scientifica che fu una delle nostre glorie nel passato e che a maggior ragione deve per l'avvenire assicurarci il posto che ci spetta fra le altre nazioni tecnicamente più progredite.

Ora è facile convincersi che queste due finalità non possono agevolmente conciliarsi fra loro.

Basta pensare che la preparazione professionale degli allievi riuscirà tanto più sicuramente quanto più presto questi verranno posti a contatto col mondo tecnico ed industriale, quanto più presto si abitueranno a vedere il lato pratico delle cose, ed a cercare in ogni teoria l'applicazione concreta, in ogni ritrovato l'utilità immediata.

Ma, così facendo, gli allievi vengono a formarsi una mentalità così prevalentemente utilitaria da non sentir più la suggestione del sapere come fine a sè stesso.

Inoltre essi non trovano più nelle nostre scuole l'ambiente ch'era nelle tradizioni universitarie più belle: l'ambiente elevato e sereno nel quale quasi inconsciamente si imparava ad amare la scienza ed a sentirne l'intima bellezza ed il fascino arcano.

Oggi alla maggioranza dei nostri giovani, non esclusi i più intraprendenti ed i meglio dotati, non viene neppure più in mente che la vita scientifica valga la pena di essere vissuta. 
Un indice espressivo della disastrosa situazione a cui ci stiamo avviando in quest'ordine di fatti ci ̀̀ offerto dallo scarso numero dei candidati che si presentano ai concorsi per cattedre di materie tecniche fondamentali, ed anche - diciamolo pure, perchè è pur troppo vero - dallo scarso valore della maggior parte dei concorrenti; scarsità di numero e di valore a spiegare la quale non basta l'esiguità, del resto innegabile, delle retribuzioni che lo Stato offre a chi alla scienza ed all'insegnamento dedica tutte le sue energie.

E si badi bene che, sotto questo punto di vista, il fenomeno dovrebbe allarmare non soltanto gli studiosi di scienze tecniche, ma anche i tecnici propriamente detti, non foss'altro per la ripercussione che esso necessariamente avrà, a non lunga scadenza, sulla costituzione media dei corpi insegnanti délle nostre scuole, e quindi sulla preparazione della gran massa degli ingegneri futuri.

Dovremmo esser tutti d'accordo nel riconoscere la necessità di prontamente ricostituire, adattandoli alle odierne condizioni di vita e di studio, quei centri di cultura superiore scientifico tecnica che, per l'avvenuto distacco delle scuole di ingegneria dalle facoltà di scienze, e pel sempre crescente predominare di incalzanti esigenze professionali, sono rimasti in questi ultimi tempi quasi completamente sacrificati.

E qui mi si consenta di chiarir bene il mio pensiero, perchè non vorrei esser frainteso.

Quando io mi riferisco al distacco delle scuole dalle facoltà come ad una delle cause che contribuirono a sacrificare gli. interessi della pura cultura, non intendo nè assumere la posa del tradizionale laudator temporis acti, nè tanto meno indicare come rimedio un ritorno all'antico; che anzi mi piace affermare esplicitamente che il cammino percorso dalle nostre scuole sulla via di una sempre crescente autonomia non fu percorso invano, che esso ha dato i suoi frutti tutt'altro che disprezzabili, e che altri anche maggiori potrà darne in avvenire. 
E d'altra parte quando io parlo dell'influenza che le esigenze professionali hanno esercitato sui nostri più attivi centri di cultura superiore, non intendo soltanto alludere alla prevalenza assunta dai corsi di intonazione pratica nelle scuole di ingegneria, ma intendo comprendervi anche l'azione deprimente che sulle stesse facoltà di scienze hanno avuto, tanto l'esodo di molti ottimi allievi ormai attratti irresistibilmente dai vantaggi materiali e morali della professione di ingegnere, quanto l'affluenza di molte, di troppe allieve allettate, più che dalle astratte speculazioni matematiche, dalla speranza di una modesta ma sicura carriera nell'insegnamento secondario.

Il che mette oggi i nostri più eletti cultori di matematiche superiori nel bivio amaro di abbassare deplorevolmente il livello scientifico dei loro insegnamenti, ovvero di trascinarsi dietro senza pietà, attraverso gli spazii ad $n$ dimensioni, una gentile schiera di signorine le quali non hanno altra aspirazione che di addestrare le nuove generazioni nei primi elementi dell'aritmetica, ovvero nelle verità più ovvie della geometria euclidea.

Ad un simile stato di cose, che va rapidamente diventando intollerabile, le facoltà stanno per opporre un radicale rimedio: l'istituzione di una laurea didattica, cioè di semplice abilitazione all' insegnamento secondario.

E sta bene! ma bisognerà pure che esse pensino a dare nuova vita alla laurea scientifica che altrimenti rischierebbe di rimanere completamente negletta.

Ed ecco dove a me pare che i bisogni delle facoltà di scienze collimino colle aspirazioni degli studiosi di scienze tecniche, rendendo possibile, e proficua, una nuovissima intesa.

Naturalmente non è attraverso gli ordinamenti ideati in tempi ormai lontani ed in relazione a situazioni oggi superate che si può pensare di raggiungere lo scopo.

« Le facoltà attuali, fra loro nettamente divise, sono ordinate secondo piani fissi ed immutabili di studii, ed hanno 
finalità proprie e determinate, finalità, è bene rilevarlo subito, professionali e scientifiche insieme, perchè, come è detto espressamente dalla stessa legge fondamentale sull'istruzione in Italia, 1'istruzione superiore ha per fine di indirizzare la gioventù, già fornita delle cognizioni generali che si acquistano nell'insegnamento secondario, agli uffici ed alle professioni per le quali si richiedono accurati studii speciali, e di mantenere ed accrescere nelle diverse parti dello Stato la cultura scientifica e letteraria.

«Ma questa divisione di facoltà si è rivelata in pratica per molti riguardi dannosa.

«Alcuni ordini di studi, come i filosofici, i geografici, gli storici, i biologici, gli economici e statistici, sono con l'attuale ordinamento sacrificati, in quanto nè i professori si possono giovare dei contatti reciproci, nè gli studenti, pur desiderandolo, possono coltivare tutte le discipline di un dato ordine di studii, secondo un programma veramente organico e scientifico.

« Lo studioso è obbligato a seguire materie per lui di nessuna utilità, e sacrificarne altre che per· lui sarebbero essenziali.

« d' uopo quindi rompere le barriere attuali fra lo facoltà e consentire di riunire, affratellare liberamente negli studii le scienze affini, spesso divise fra facoltà diverse, senza obbligo di seguire piani prestabiliti di studii ».

Con questa bella motivazione, che io ho voluto riportare per disteso dagli atti del Consiglio superiore dell'istruzione pubblica, quest'alto consesso faceva suo, or son due anni, un antico voto per la libertà degli studii superiori, formulato più nettamente nei recenti congressi universitarii dell'aprile 1912 e del novembre 1916; ed il voto ha avuto il suo compimento in due decreti luogotenenziali, l'uno del 28 ottobre $1917 \mathrm{di}$ iniziativa del ministro Ruffini, l'altro del 3 febbraio 1918 dovuto al ministro Berenini.

Questi decreti permettono di « conferire un titolo accademico a tutti coloro che intendono perseguire un puro ideale 
scientifico, ovvero educarsi scientificamente per un indirizzo. professionale che non corrisponda a quelli disciplinati dalla legge e dai regolamenti attuali e muniti di effetti legali dallo Stato » [relazione di S. E. Ruffini in data 2 giugno 1917].

Dal punto di vista professionale ciò faciliterà le specializzazioni nelle tante applicazioni pratiche delle scienze sperimentali, e la conseguente formazione degli specialisti, in particolare di quelli per cui sono superflue ovvero insufficienti le lauree attuali.

Ben maggiore importanza l'innovazione avrà poi dal puntodi vista scientifico, poichè consentirà da un lato di dare la dovuta dignita a materie oggi trascurate, mentre dall'altro lato permetterd di soddisfare a tutte le vocazioni ed a tutti i bisogni di cultura superiore che nella vita della nazione potessero manifestarsi.

Ciò posto ritorniamo al caso specifico delle facoltà di scienze e delle scuole di ingegneria.

Si rileva subito la grande varietà ed elasticità di ordinamenti che i nuovi decreti sulle lauree scientifiche permetteranno di dare a quel centro di studii matematici che dovrebbe prosperare accanto al progettato corso per la laurea in matematica a scopo semplicemente didattico.

Basta por mente alla più ampia facoltà che lo studente verrà ad avere di concentrare attorno a poche, o anche ad una sola disciplina, la maggior e miglior parte della sua attività, iscrivendosi ripetutamente alla stessa materia, sia contemporaneamente presso diversi insegnanti - insegnanti ufficiali o docenti liberi - sia successivamente nei diversi anni presso lo stesso insegnante, quando lo svolgimento che questi dà al suo corso sia diverso nella sostanza o nell' in. dirizzo.

Al sano criterio delle autorità accademiche è affidato dal legislatore il compito delicato di giudicare della sufficienzid della durata degli studii compiuti da ciascun allievo e del numero e qualità dei corsi da lui scelti per ottenere la laurea 
cui aspira; ed esse certo si varranno di questa facoltà per esercitare un azione sapientemente moderatrice, $\mathrm{e}$ in particolare per porre un giusto freno a tendenze di specializzazione eccessive.

Tuttavia non è azzardato prevedere come probabile una pronta differenziazione della laurea in scienze matematiche propriamente detta, che continuerà ad imperniarsi sui corsi di analisi superiore, di geometria superiore e di fisica matematica da una laurea in scienze astronomiche per la quale verranno posti nel dovuto rilievo i corsi di geodesia teoretica, di meccanica celeste e di astronomia.

Ma non basta. Sono le scuole di ingegneria quelle che possono e debbono portare a quest' opera di rinnovamento il più valido impulso, prendendo l'iniziativa perchè nei nostri maggiori centri di studi superiori, sia reso possibile ai giovani il conseguimento di una laurea in scienze tecniche, la cui fisionomia può essere facilmente prevista, almeno nelle sue linee generali.

To ho avuto occasione non soltanto di studiare il problema nell' interesse di qualche mio allievo, ma anche di discuterlo ampiamente coi miei colleghi dell' Università di Pisa, presso la quale un primo, sia pur modesto, tentativo sta per venire in tal senso effettuato. $\mathrm{E}$ credo di poter fissare $\mathrm{i}$ seguenti punti fondamentali dai quali difficilmente in pratica ci si potrà scostare.

Il corso sarà almeno quadriennale, con un primo biennio di carattere propedeutico non dissimile da quello che oggi frequentano gli aspiranti alle lauree in matematica ed in fisica e gli allievi ingegneri (meccanica razionale inclusa).

Negli anni successivi le matematiche superiori, le meccaniche applicate $\theta$ le fisiche tecniche dovrebbero essere in giuste proporzioni rappresentate: le prime da un corso di analisi e da almeno un corso di fisica matematica; le seconde da corsi di teoria dell'elasticità, di dinamica applicata, di dinamica dei fluidi; le ultime da corsi di termotecnica e di elettrotecnica. 
Se sia utile che questi corsi siano puramente e semplicemente quelli che con pari titolo si impartiscono rispettiva. mente nelle facoltà di scienze e nelle scuole di ingegneria, ̀̀ difficile dire in modo assoluto; certo questa sarà la soluzione immediata del problema - tanto più che i citati decreti nou prevedono l'istituzione di corsi ad hoc - e spetterà al criterio personale di ciascun insegnante l'accentuare od il moderare, a seconda dei casi, le sue esigenze nei riguardi degli aspiranti alla laura in scienze tecniche.

E d'altronde da prevedersi che in un primo tempo questa, come del resto quasi tutte le lauree speciali, verrà richiesta soltanto a titolo di perfezionamento da giovani che avranno già preventivamente conseguita una delle lauree attuali.

Ad essi i decreti che ho sopra citati concedono il conseguimento della laurea speciale in un solo anno di studio, colla frequenza di anche solo tre corsi.

Questa disposizione, assai equa del resto, permettera di tentare l'innovazione anche là dove potrebbe sembrar difficile una soddisfacente realizzazione immediata del corso completo come ente per sè stante.

Il tempo farà il resto: cioè permetterà a noi di portare gradualmente i nuovi centri di studii al desiderato grado di sviluppo e di perfezione, istituendo anche, ove sia possibile, qualche corso nuovo con indirizzo al nuovo scopo più specialmente adattato; e convincera i migliori fra i giovani - che non abbiano immediate mire professionali - ad avviarsi fidu. ciosi per le nuove vie loro aperte da sì ben intesa libertà di ordinamenti scolastici, affidando il loro avvenire più alla sincerita delle loro vocazioni che non alle maggiori o minori garanzie annesse ad un diploma di Stato.

In ogni caso un grande vantaggio si sarà fin d'ora assicurato alle scuole di ingegneria, poichè esse, partecipando nella misura delle loro forze e delle loro competenze alla formazione di questi nuovi centri di studi tecnici superiori, potranno a buon diritto ritenersi dispensate dall'obbligo di 
ulteriormente subordinare i loro ordinamenti interni a finalità puramente scientifiche o comunque non strettamente professionali.

Ed il problema della riforma di queste scuole secondo direttive ispirate alle più strette esigenze della vita professionale e dell'industria verrebbe ad essere grandemente facilitato; ed il maggiore ostacolo che oggi si frapponga alla sua risoluzione resterebbe eliminato per sempre.

Voltaggio, agosto 1919. 
AtTilio Marioti, gerente responsabile. 